\title{
Die Kinetik der thermischen Reaktion zwisehen Pentafluorschwefelhypofluorid und Kohlenmonoxid
}

\author{
Von \\ A. J. Colussi und H. J. SChUMacher \\ Instituto Superior de Investigaciones, Facultad de Ciencias Exactas \\ Universidad Nacional de la Plata, La Plata, Argentinien
}

(Eingegangen am 8. April 1970)

Die Reaktion zwischen $\mathrm{SF}_{5} \mathrm{OF}$ und $\mathrm{CO}$ wurde in einem Quarzgefä $\beta$ bei Temperaturen zwischen 110 und $150^{\circ} \mathrm{C}$ untersucht. Sie verläuft praktisch ohne Druckänderung und es werden neben Spuren von $\mathrm{COF}_{2}$ in nahezu quantitativer Weise äquimolekulare Mengen von $\mathrm{SF}_{6}$ und $\mathrm{CO}_{2}$ gebildet.

$$
\mathrm{SF}_{5} \mathrm{OF}+\mathrm{CO} \stackrel{\text { Quarz }}{\longrightarrow} \mathrm{SF}_{6}+\mathrm{CO}_{2} .
$$

Der Reaktionsablauf wird vom Verhältnis Oberfläche zu Volumen des Reaktionsgefäßes nicht beeinflußt. Thre Geschwindigkeit verläuft proportional mit der Wurzel aus der Konzentration des $C O$ und der $3 / 2$ ten Potenz der Konzentration des $\mathrm{SF}_{5} \mathrm{OF}$. Der Gesamtdruck, wie auch inerte Zusatzgase haben einen sehr geringen, die Reaktionsprodukte keinerlei Einfluß auf die Reaktion.

$$
\mathrm{I}+\frac{d\left|S F_{6}\right|}{d t}=k\left|S F_{5} O F\right|^{3 / 2}|C O|^{1 / 2} .
$$

Die Reaktion ist ohne Zweifel homogener Natur und verläuft über eine Kette von mäßiger Länge.

Selbst geringe Mengen von $\mathrm{O}_{2}$ setzen die Geschwindigkeit auf etwas mehr als die Hälfte ihres ursprünglichen Wertes herab. Unter den Reaktionsprodukten treten nunmehr geringe Mengen des Peroxides, $(\mathrm{COF})_{2} \mathrm{O}_{2}$ auf. Für das sauerstofffreie System wurde folgendes Reaktionsschema aufgestellt:

$$
\begin{aligned}
S F_{5} O F^{\prime}+C O & =S F_{5} O^{*}+C O F^{*} \\
S F_{5} O^{*}+C O & =S F_{5}^{*}+\underline{C O_{2}} \\
S F_{5}^{*}+S F_{5} O F & =\underline{S F_{6}}+S F_{5} O^{*} \\
C O F^{*}+S F_{5} O F & =C O F_{2}+S F_{5} O^{*} \\
S F_{5}^{*}+S F_{5}^{*} & =S F_{10},
\end{aligned}
$$


hieraus folgt:

$$
\mathrm{II}+\frac{d\left|S F_{6}\right|}{d t}=+\frac{d\left|C O_{2}\right|}{d t}=2 k_{3}\left(k_{1} / k_{5}\right)^{1 / 2}\left|S F_{5} O F\right|^{3 / 2}|C O|^{1 / 2} .
$$

Mit $k=2 k_{3}\left(k_{1} / k_{5}\right)^{1 / 2}$ geht Gl. (I) in Gl. (II) über. Aus dem Temperaturkoeffizienten von $k$ ergibt sich:

$$
q=18 \pm 1 \mathrm{keal}=q_{3}+\frac{1}{2} q_{1}-\frac{1}{2} q_{5} .
$$

Für $q_{5}=0$ und unter der Annahme, daß $q_{3}$ von der Größenordnung einiger keal, etwa 4 kcal, ist, ergibt sich $q_{1}=28 \pm 3$ kcal.

Die Sauerstoffhemmung wird im wesentlichen durch die schnelle Reaktion der nach (1) gebildeten COF-Radikale mit $\mathrm{O}_{2}$ hervorgerufen, wodurch die Geschwindigkeit der Gesamtreaktion auf die Hälfte reduziert wird.

In $N i$ - und $C u$-Gefäßen wird eine sehr viel schnellere Geschwindigkeit als in solchen aus Quarz beobachtet. Es bilden sich nunmehr in Übereinstimmung mit Beobachtungen von CADY und WrLIAMson äquimolekulare Mengen von $\mathrm{SOF}_{4}$ und $\mathrm{COF}_{2}$. Die Reaktionen in diesen Gefäßen sind heterogener Natur.

$$
\mathrm{SF}_{5} \mathrm{OF}+\mathrm{CO} \stackrel{\mathrm{Ni}, \mathrm{Cu}}{\longrightarrow} \mathrm{COF}_{2}+\mathrm{SOF}_{4} \quad \text { (heterogene Reaktion). }
$$

Wrutramson und CADY ${ }^{1}$ berichteten vor einiger Zeit, daß sich Pentafluorschwefelhypofluorid und Kohlenmonoxid quantitativ zu Thionyltetrafluorid und Fluorphosgen umsetzen. Sie hatten bei ihren Versuchen Nickel- und Kupfer-Reaktoren verwendet und im ersteren Fall bei Temperaturen zwischen 20 und $160^{\circ} \mathrm{C}$ und Drucken von etwa 100 Torr und im zweiten Fall bei Zimmertemperatur und einem Druck von etwa fünf Atmosphären gearbeitet.

Um den Mechanismus dieser Reaktion aufzuklären, haben wir ihre Kinetik einem eingehenden Studium unterzogen.

\section{Experimenteller Teil}

Da zu erwarten war, daß die Reaktion ohne Molzahländerung verlief, sollte ihr Verlauf in der Weise verfolgt werden, daß sie nach einer bestimmten Zeit durch möglichst schnelles Abkühlen unterbrochen und sodann die Konzentration eines oder mehrerer der Reaktionsteilnehmer in geeigneter Weise bestimmt wurde. Es wurde hierzu eine Apparatur verwendet, die sich bei früheren Arbeiten ${ }^{2}$ bewährt hatte. Da Pentafluorschwefelhypofluorid bei den zu erwartenden Reaktionstemperaturen gegenüber Quarz inert ist, wurde als Reaktionsgefäß eine Quarzkugel von $346 \mathrm{ml}$ Inhalt gewählt.

1 S. M. Williamson und G. H. Cady, Inorg. Chem. 1 (1962) 673.

2 E. J. Vasini und H. J. Schumacher, Z. physik. Chem. Neue Folge 65 (1969) 238. 


\section{Die verwendeten Gase}

$S F_{5} O F$ : Es wurde nach dem Verfahren von RuFF und Lustig ${ }^{3}$ durch Reaktion von Fluor mit Thionylfluorid in Gegenwart eines Überschusses von Cäsiumfluorid bei Temperaturen von etwa $100^{\circ} \mathrm{C}$ in einem gut fluorierten Nickelgefäß dargestellt und nach mehrmaligem Destillieren bei tiefen Temperaturen rein erhalten. Sein Reinheitsgrad wurde durch Aufnahmen des IR-Spektrums, Dampfdruckmessungen und chemische Analyse überprüft und sichergestellt.

$C O:$ Es wurde von der Firma Air Products and Chemicals bezogen und zur Entfernung jeglichen etwa vorhandenen Sauerstoffs über auf etwa $500{ }^{\circ} \mathrm{C}$ erhitzten Kupferschwamm geleitet.

$\mathrm{O}_{2}, \mathrm{~N}_{2}, \mathrm{CO}_{2}$ und $\mathrm{CF}_{4}$ waren käufliche Produkte. Sie wurden nach konventionellen Methoden gereinigt und ebenso, wie das $C O$, in 2-1Kolben aufbewahrt.

\section{Die Versuche und ihre Ergebnisse}

Die Versuche wurden stets in der Weise durchgeführt, daß das im Unterschuß vorhandene Reaktionsgas als erstes in das gut evakuierte Reaktionsgefäß eintrat. Sodann folgte der zweite Reaktionsteilnehmer und gegebenenfalls das Zusatzgas. Nach einer bestimmten, variablen Zeit wurde der Thermostat schnell gesenkt und das Reaktionsgefäß in ein mit flüssiger Luft gefülltes Dewar getaucht. Hierauf wurde das überschüssige Kohlenmonoxid durch Abpumpen entfernt. Bei bekannter Stöchiometrie der Reaktion konnte aus dem Druck der Restgase bei Versuchstemperatur der Umsatz berechnet werden.

Da die mit dem „Abschrecken“ der Reaktion verbundenen Manipulationen eine gewisse Zeit - etwa 10-12 Sekunden - erforderten, wurden die Reaktionszeiten stets so groß gehalten, daß die hierdurch bedingte Ungenauigkeit in der Bestimmung der Reaktionszeit ohne Bedeutung war. Der Umsatz bei den einzelnen Versuchen lag im allgemeinen zwischen 10 und $30 \%$, bei einigen wenigen betrug er $40 \%$.

Vorversuche hatten gezeigt, daß die Reaktion bei Temperaturen um $130^{\circ} \mathrm{C}$ mit bequem meßbarer Geschwindigkeit verläuft und daß bis zum Reaktionsende keine merkliche Druckänderung auftritt. In den IR-Spektren der Produkte konnten, abgesehen von den mit sehr geringer Intensität auftretenden stärksten Banden des Fluorphosgens, lediglich die Banden des Fluorhexafluorids und des Kohlendioxids beobachtet werden. Die Produkte der Reaktion sind also nicht

\footnotetext{
${ }^{3}$ J. K. Ruff und M. Lustig, Inorg. Chem. 3 (1964) 1422.
} 
Thionyltetrafluorid und Fluorsphosgen - letzteres trat nur in Spuren auf -, sondern Fluorhexafluorid und Kohlendioxid.

$$
\mathrm{SF}_{5} \mathrm{OF}+\mathrm{CO} \stackrel{\text { Quarz }}{\longrightarrow} \mathrm{SF}_{6}+\mathrm{CO}_{2} .
$$

Im Laufe der Untersuchungen wurde von Zeit zu Zeit die Zusammensetzung der Reaktionsprodukte durch Aufnahme ihrer IRSpektren überprüft. Diese Aufnahmen wurden mit einem PerkinElmer-221-Spektrometer im $\mathrm{NaCl}$-Gebiet ausgeführt. Es wurden stets die gleichen Ergebnisse erhalten.

Die eigentlichen Versuche wurden bei 110,130 und $150^{\circ} \mathrm{C}$ durchgeführt. Hierbei wurden die Anfangsdrucke des Pentafluorschwefelhypofluorids zwischen 15 und 150 Torr und die des Kohlenmonoxid zwischen 30 und 250 Torr variiert. Es wurden ferner eine Anzahl von Versuchen unter Zusatz größerer Mengen von Stickstoff und Kohlendioxid und wechselnder Mengen von Sauerstoff gemacht. Um die Homogenität der Reaktion zu überprüfen, wurden schließlich eine Reihe von Versuchen in einem Quarzgefäß mit relativ großer Oberfläche durchgeführt. Als Reaktiongefäß diente in diesem Fall eine Quarzkugel, die mit Quarzröhrchen von etwa $1 \mathrm{~cm}$ Länge mit $0,6 \mathrm{~cm}$ Durchmesser gefüllt war. Das Verhältnis von Oberfläche zu Volumen war in diesem Gefäß etwa um den Faktor 5 größer als in dem ursprünglichen.

Alle Versuche waren innerhalb der durch die Versuchstechnik bedingten nicht sehr hohen Genauigkeit - die einzelnen Meßwerte streuten um etwa $10 \%$ um den Mittelwert - gut reproduzierbar. Es wurde offenbar, daß die Gefäßwand keinerlei „Einfluß" auf den Reaktionsablauf und die Reaktionsgeschwindigkeit hatte. Es kann somit als sicher angesehen werden, daß die hier untersuchte Reaktion eine homogene Gasreaktion ist.

Die Versuche zeigten weiterhin, daß die Reaktion keine Induktionsperiode besaß und daß ihre Geschwindigkeit proportional mit der Wurzel aus der Konzentration des Kohlenmonoxides und der 1,5 ten Potenz der Konzentration des Pentafluorschwefelhypofluorids verlief. Die Reaktionsprodukte übten keinen, große Mengen zugesetzter, inerter Gase einen sehr geringen, geschwindigkeitsvermindernden Einfluß aus. Die Geschwindigkeit kann somit durch folgende Gleichung dargestellt werden:

$$
\begin{aligned}
A-\frac{d[\mathrm{CO}]}{d t} & =-\frac{d\left[S F_{5} O F\right]}{d t}=+\frac{d\left[S F_{6}\right]}{d t}=+\frac{d\left[\mathrm{CO}_{2}\right]}{d t} \\
& =k\left[S F_{5} O F\right]^{3 / 2}[C O]^{1 / 2} .
\end{aligned}
$$


Sauerstoff hat eine charakteristische, hemmende Wirkung. Bereits so geringe Mengen, wie ein Torr, setzen die Geschwindigkeit auf etwas mehr als die Hälfte ihres ursprüngliches Wertes herab. Die Anwesenheit großer Sauerstoffmengen verstärkt diesen Effekt nicht. Die Geschwindigkeit liegt stets zwischen 0,3 und 0,4 ihres ,Normalwertes". Die Reaktionsprodukte bleiben bei den Versuchen mit Sauerstoff die gleichen. Abgesehen von geringen Mengen des Peroxides $(\mathrm{COF})_{2} \mathrm{O}_{2}$, das sehr leicht durch sein IR-Spektrum zu erkennen ist, konnten keine neuen Substanzen nachgewiesen werden. Die in den sauerstofffreien Versuchen auftretenden an sich schon sehr geringen Mengen von Fluorphosgen waren in den bei Gegenwart von Sauerstoff durchgeführten nahezu völlig verschwunden.

Da CADY und Mitarbeiter, die mit Nickel und Kupferreaktoren gearbeitet hatten, eine quantitative Bildung von Thionyltetrafluorid und Fluorphosgen beobachtet hatten, wurden von uns auch einige Versuche in einem Nickelgefäß durchgeführt. Wir konnten feststellen, daß die Reaktion zwar ebenfalls ohne Molzahländerung vonstatten geht, daß ihre Geschwindigkeit jedoch um mehrere Größenordnungen höher liegt als in Quarzgefäßen. Als Reaktionsprodukte traten nunmehr tatsächlich äquimolekulare Mengen von Thionyltetrafluorid und Fluorphosgen auf. Auf Grund dieser Daten kann mit Sicherheit geschlossen werden, daß die Reaktion in $\mathrm{Ni}$ - und $\mathrm{Cu}$-Gefäßen als heterogene Wandreaktion abläuft. Wir haben daraufhin diese Reaktion nicht weiter untersucht:

$$
\mathrm{SF}_{5} \mathrm{OF}+\mathrm{CO} \stackrel{\mathrm{Cu}, \mathrm{Ni}}{\longrightarrow} \mathrm{SOF}_{4}+\mathrm{COF}_{2} \text { (heterogene Reaktion). }
$$

Die im Verlaufe unserer Versuche erhaltenen Ergebnisse sind in den folgenden Tabellen zusammengestellt. Es bedeuten hierin $N_{r}$ die Versuchsnummer, $T$ die Temperatur in Grad Celsius, $\Delta t$ die Versuchszeit in Minuten, und $\Delta p$ den dieser Zeit entsprechenden Umsatz. $S_{5} O F, C O, X$ sind die Anfangsdrucke der entsprechenden Gase in Torr bei Versuchstemperatur und $k$ ist die mit Hilfe der integrierten Form der Gleichung $(A)$ berechnete Geschwindigkeitkonstante:

$$
k=\frac{1}{\Delta t} \cdot \frac{2}{a-b}\left[\left(\frac{b-x}{a-x}\right)^{1 / 2}-\left(\frac{b}{a}\right)^{1 / 2}\right] .
$$

In dieser Gleichung bedeuten $a$ und $b$ die Anfangsdrucke der Reaktionsteilnehmer und $x$ den Umsatz. 
Tabelle $1.110^{\circ} \mathrm{C}$

\begin{tabular}{l|r|r|r|r|r|c}
\hline$N_{v}$ & \multicolumn{1}{|c|}{$\Delta t_{\min }$} & $\Delta p_{\text {Torr }}$ & $S F_{5}$ OF & $C O$ & $X$ & $\begin{array}{c}k \cdot 10^{4} \\
\text { Torr }^{-1} \text { min }^{-1}\end{array}$ \\
\hline 33 & 60,02 & 6,0 & 30,2 & 50,3 & - & 1,03 \\
34 & 22,12 & 6,1 & 60,0 & 50,4 & - & 0,85 \\
35 & 11,55 & 5,8 & 90,3 & 50,2 & - & 0,91 \\
36 & 42,75 & 5,7 & 30,1 & 100,1 & - & 0,96 \\
38 & 7,90 & 7,9 & 150,2 & 49,9 & - & 0,83 \\
39 & 26,82 & 5,9 & 30,0 & 250,0 & - & 1,00 \\
45 & 167,56 & 5,2 & 15,0 & 50,4 & - & 1,06 \\
54 & 62,13 & 5,9 & 30,3 & 50,1 & - & 0,98 \\
67 & 60,16 & 11,4 & 50,0 & 50,2 & - & 0,83 \\
69 & 60,00 & 11,5 & 50,0 & 50,1 & - & $0,83 *$ \\
& & & & & &
\end{tabular}

$\bar{k}=0,928 \cdot 10^{-4} \mathrm{Torr}^{-1} \mathrm{~min}^{-1}$

\begin{tabular}{l|r|l|l|l|l|l}
\hline 47 & 61,05 & 4,9 & 29,7 & 50,0 & $N_{2}=233,3$ Torr & 0,81 \\
48 & 59,53 & 5,2 & 30,3 & 49,9 & $N_{2}=139,7$ Torr & 0,87 \\
49 & 61,43 & 4,3 & 30,0 & 50,2 & $N_{2}=306,0$ Torr & 0,70 \\
56 & 60,00 & 4,6 & 25,5 & 49,8 & $C F_{4}=96,2$ Torr & 1,00 \\
57 & 169,93 & 4,2 & 29,9 & 12,3 & $C F_{4}=160,6$ Torr & 0,70
\end{tabular}

* Oberflächengefäß.

Tabelle 2. $130^{\circ} \mathrm{C}$

\begin{tabular}{r|r|r|r|r|r|c}
\hline$N_{v}$ & $\Delta t_{\min }$ & $\Delta p_{\text {Torr }}$ & $S F_{5} O F$ & $C O$ & $X$ & $\begin{array}{c}k \cdot 10^{4} \\
\text { Torr }^{-1} \text { min }^{-1}\end{array}$ \\
\hline 12 & 20,32 & 2,8 & 23,5 & 30,5 & - & 2,39 \\
14 & 21,03 & 4,8 & 25,0 & 50,1 & - & 3,07 \\
17 & 39,92 & 7,2 & 25,2 & 50,3 & - & 2,67 \\
18 & 39,75 & 7,4 & 24,9 & 50,1 & - & 2,85 \\
19 & 19,66 & 3,9 & 25,1 & 49,9 & - & 2,58 \\
20 & 10,05 & 5,1 & 25,2 & 249,8 & - & 3,01 \\
21 & 10,75 & 5,1 & 39,8 & 49,7 & - & 3,01 \\
22 & 80,25 & 11,3 & 24,8 & 50,3 & - & 2,69 \\
23 & 8,00 & 8,6 & 80,0 & 50,0 & - & 2,50 \\
25 & 69,96 & 5,0 & 14,8 & 50,4 & - & 2,46 \\
63 & 40,05 & 7,6 & 24,9 & 50,1 & - & 2,93 \\
& & & & & &
\end{tabular}

$\bar{k}=2.74 \cdot 10^{-4}$ Torr $^{-1} \min ^{-1}$

\begin{tabular}{l|l|l|l|l|l|l}
28 & 40,04 & 6,7 & 24,8 & 49,7 & $C O_{2}=211,0$ Torr & 2,53 \\
29 & 40,08 & 5,4 & 24,7 & 49,9 & $C O_{2}=211,3$ Torr & 1,94
\end{tabular}


Tabelle $3.150^{\circ} \mathrm{C}$

\begin{tabular}{r|r|r|r|r|r|r}
\hline \multicolumn{1}{|c|}{$N_{v}$} & \multicolumn{1}{|c|}{$\Delta t_{\min }$} & $\Delta p_{\text {Torr }}$ & $S F_{5}$ OF & $C O$ & $X$ & $\begin{array}{c}k \cdot 10^{4} \\
\text { Torr }^{-1} \text { min }^{-1}\end{array}$ \\
\hline 8 & 13,73 & 6,9 & 23,4 & 60,0 & - & 7,68 \\
9 & 10,52 & 8,4 & 23,4 & 120,2 & - & 9,10 \\
10 & 6,90 & 7,4 & 46,8 & 30,0 & - & 7,42 \\
40 & 20,03 & 9,9 & 25,6 & 40,1 & - & 9,36 \\
41 & 10,24 & 5,9 & 25,4 & 40,0 & - & 9,09 \\
42 & 21,90 & 5,3 & 15,0 & 40,1 & - & 9,42 \\
43 & 7,74 & 6,4 & 25,1 & 80,5 & - & 9,33 \\
44 & 6,81 & 8,6 & 40,0 & 40,2 & - & 7,34 \\
\end{tabular}

$\bar{k}=8,59 \cdot 10^{-4}$ Torr $^{-1} \min ^{-1}$

Tabelle 4. Versuche mit $\mathrm{O}_{2}$-Zusatz

\begin{tabular}{r|r|r|r|r|r|r}
\hline$N_{v}$ & $T^{\circ} C$ & $\Delta t_{\min }$ & $\Delta p_{\text {Torr }}$ & $S F_{5} O F$ & $C O$ & $O_{2}$ \\
\hline 33 & 110 & 60,02 & 6,0 & 30,2 & 50,3 & - \\
51 & 110 & 60,64 & 1,9 & 29,9 & 50,2 & 100,0 \\
52 & 110 & 62,26 & 1,8 & 29,8 & 50,0 & 40,2 \\
53 & 110 & 60,45 & 1,8 & 29,8 & 50,1 & 10,1 \\
55 & 110 & 61,62 & 1,8 & 30,1 & 50,1 & 2,8 \\
66 & 110 & 239,85 & 5,6 & 29,8 & 50,5 & 20,5 \\
67 & 110 & 60,16 & 11,4 & 50,0 & 50,1 & - \\
68 & 110 & 60,87 & 3,5 & 49,8 & 49,9 & 14,2 \\
70 & 110 & 60,00 & 2,9 & 49,9 & 50,1 & $20,0^{*}$ \\
71 & 110 & 60,20 & 2,8 & 49,9 & 50,0 & $20,2^{*}$ \\
72 & 110 & 245,70 & 10,4 & 50,0 & 50,0 & $20,2^{*}$ \\
73 & 110 & 245,20 & 10,1 & 50,3 & 50,2 & $11,5^{*}$ \\
63 & 130 & 40,05 & 7,6 & 24,9 & 50,1 & - \\
61 & 130 & 40,50 & 3,3 & 24,8 & 50,2 & 3,6 \\
62 & 130 & 39,93 & 3,2 & 24,9 & 50,1 & 12,8 \\
64 & 130 & 39,97 & 3,4 & 24,9 & 50,2 & 48,5 \\
65 & 150 & 21,19 & 3,4 & 23,5 & 30,0 & 60,0
\end{tabular}

* Oberflächengefäß.

Als Mittelwerte der Konstanten für die verschiedenen Temperaturen wurden folgende Zahlen gefunden: $k_{110}=0,928 \cdot 10^{-4}, k_{130}=$ $2,74 \cdot 10^{-4}, k_{150}=8,59 \cdot 10^{-4}$ Torr $^{-1} \mathrm{~min}^{-1}$ oder umgerechnet auf Sekunden und $\mathrm{Mol} / \mathrm{l}: \quad k_{110}=4,08 \cdot 10^{-2}, \quad k_{130}=11,47 \cdot 10^{-2}, \quad k_{150}=$ $39,52 \cdot 10^{-2} \mathrm{l} \mathrm{Mol}^{-1} \mathrm{sec}^{-1}$. Aus diesen Konstanten errechnet sich eine scheinbare Aktivierungsenergie von $q=18 \pm 1 \mathrm{kcal}$. 


\section{Diskussion und Ergebnisse}

Aus den Ergebnissen folgt in einwandfreier Weise, daß die Reaktion in Quarzgefäßen als homogene Gasreaktion abläuft. Die Form der Geschwindigkeitsgleichung zeigt an, daß es sich um eine Kettenreaktion handelt. Aus dem allgemeinen Verhalten der Reaktion kann ferner geschlossen werden, daß die Kettenlänge unter unseren Versuchsbedingungen nur von mäßiger Größe ist. Da die Gefäßwand keinen, der Gesamtdruck und zugesetzte inerte Gase nur einen sehr geringen Einfluß auf den Reaktionablauf ausüben, kann gefolgert werden, daß im Reaktionsschema weder Desaktivierungsprozesse energiereicher Moleküle noch Dreierstoßreaktionen auftreten.

Der Primärprozeß dürfte eine bimolekulare Reaktion zwischen den Ausgangsmolekülen sein, bei der die beiden Radikale $\mathrm{SF}_{5} \mathrm{O}$ und $\mathrm{FCO}$ entstehen. Da die $O-F$-Bindung sehr viel schwächer als die $C-F$ Bindung ist, kann angenommen werden, daß das $\mathrm{FCO}$ sich in schneller Reaktion mit einem Pentafluorschwefelhypofluoridmolekül unter Bildung von Fluorphosgen und einem weiteren $\mathrm{SF}_{5} \mathrm{O}$-Radikal umsetzt. Letzteres bildet durch Reaktion mit Kohlenmonoxid das als Endprodukt auftretende Kohlendioxid und setzt hierbei ein $S F_{5^{-}}$ Radikal frei, das nun seinerseits mit einem Pentafluorschwefelhypofluoridmolekül weiterreagiert, wobei unter Regeneration eines $\mathrm{SF}_{5} \mathrm{O}$ Radikals das andere Endprodukt, Schwefelhexafluorid, entsteht.

Entsprechend dem Wurzelausdruck in der Geschwindigkeitsgleichung muß der Kettenabbruch eine bimolekulare Reaktion zweier Radikale sein. Da im IR-Spektrum keine $S F_{5} O O S F_{5}$-Banden beobachtet werden konnten, nehmen wir an, daß es sich um die Reaktion zweier $S F_{5}$-Radikale handelt. Wir werden somit zu dem folgenden Reaktionsschema geführt:

$$
\begin{aligned}
S F_{5} O F+C O & =S F_{5} O+C O F \\
C O F+S F_{5} O F & =C O F_{2}+S F_{5} O \\
S F_{5} O+C O & =S F_{5}+\underline{C O}_{2} \\
S F_{5}+S F_{5} O F & =S F_{5} O+\underline{S F_{6}} \\
S F_{5}+S F_{5} & =S_{2} F_{10} .
\end{aligned}
$$

Nimmt man einen quasistationären Zustand an, so erhält man hieraus die folgende Geschwindigkeitsgleichung:

$$
\mathrm{I}+\frac{d\left[S F_{6}\right]}{d t}=+\frac{d\left[\mathrm{CO}_{2}\right]}{d t}=k_{4}\left(k_{1} / k_{5}\right)^{1 / 2}\left[S F_{5} O F\right]^{3 / 2}[C O]^{1 / 2} .
$$


Für $k=k_{4}\left(k_{1} / k_{5}\right)^{1 / 2}$ geht Gl. (1) in die experimentell gefundene Geschwindigkeitsgleichung $(A)$ über.

Dem Reaktionsschema entsprechend entstehen neben den eigentlichen Reaktionsprodukten, Schwefelhexafluorid und Kohlendioxid, der Häufigkeit des Primärprozesses entsprechend, also in sehr geringen Mengen, Fluorphosgen und Schwefeldekafluorid. Ersteres konnte durch seine charakteristischen Banden im IR nachgewiesen werden, letzteres nicht. Es ist hierbei jedoch zu berücksichtigen, daß etwa auftretende Banden des $S_{2} F_{10}$ durch die des stets in großem Überschuß auftretenden Schwefelhexafluorids verdeckt werden. Eine Abtrennung des $S_{2} F_{10}$ ist wegen der zu erwartenden geringen Mengen nicht möglich.

In der Geschwindigkeitsgleichung erscheinen lediglich die Konstanten der Reaktionen (1), (4) und (5), die alle stark exotherm sind. Es kann als sicher angesehen werden, daß die Aktivierungsenergie von (5) nahezu Null und die von (4) sehr gering, etwa von der Größenordnung einiger kcal ist - wir nehmen als Schätzwert $q_{4}=4 \mathrm{kcal}$. Unter diesen Annahmen läßt sich die Aktivierungsenergie des Primärprozesses aus der scheinbaren Aktivierungsenergie der Gesamtreaktion berechnen.

$$
q=q_{4}+\frac{1}{2} q_{1}-\frac{1}{2} q_{5}=18 \pm 1 \mathrm{kcal} \mathrm{mol}^{-1}, \text { somit } q_{1}=28 \pm 3 \mathrm{kcal} .
$$

Da die Absolutgeschwindigkeit der Primärreaktion nicht genau bestimmt werden kann, können auch für die Kettenlänge keine genauen Werte angegeben werden.

Um auf die Wirkung des Gesamtdruckes auf die Reaktion zurückzukommen, so war diese sehr gering. Einer Erhöhung des Gesamtdruckes von 100 auf 300 Torr, also um den Faktor 3, entsprach im Mittel eine Geschwindigkeitsverminderung von etwa $10 \%$, also ein Wert, der nur um ein geringes größer ist als die Versuchsungenauigkeit. Möglicherweise ist dieser Effekt durch Reaktion (5) bedingt. Hier handelt es sich zwar um die Assoziation zweier 6atomiger Radikale, aber man weiß nicht, wieviel der vorhandenen Freiheitsgrade aktiv sind, so daß eine schwache Druckabhängigkeit der Reaktion unter unseren Versuchsbedingungen nicht ausgeschlossen werden kann.

Was den Einfluß des Sauerstoffs anbetrifft, so konnte er nicht in allen Einzelheiten aufgeklärt werden. Eine Verminderung der Geschwindigkeit auf die Hälfte, bedingt durch die Anwesenheit bereits geringer Mengen von Sauerstoff, ist zwar ohne weiteres verständlich; denn man weiß, daß $C O F$-Radikale schnell mit Sauerstoff reagieren, 
wobei sich als Endprodukt das Peroxid $(\mathrm{COF})_{2} \mathrm{O}_{2}{ }^{4}$ bildet. Letzteres wurde auch in den zu erwartenden geringen Mengen durch sein charakteristisches Spektrum im IR identifiziert. Die bei den ,Sauerstoffversuchen" bisweilen beobachtete geringe Druckverminderung beruht möglicherweise auf der Bildung dieser Verbindung.

Der tatsächlich beobachtete hemmende Einfluß des Sauerstoffs war jedoch etwas größer. Die Geschwindigkeit wurde auf etwa den 0,3-ten bis 0,4-ten Teil ihres ursprünglichen Wertes herabgesetzt. Um diesen Effekt, der in seiner Höhe unabhängig von der Konzentration des anwesenden Sauerstoffs ist, zu erklären, muß angenommen werden, daß außer dem $C O F$ auch alle übrigen Kettenträger mit Sauerstoff eine Verbindung bzw. Assoziation eingehen. Da die beobachtete Hemmung zwar konstant, jedoch relativ gering ist, muß gefolgert werden, daß nur eine Bruchteil, und zwar ein konstanter Bruchteil, dieser Zwischenverbindung, die entsprechenden Kettenträger in eine inerte Verbindung überführt, während der ebenfalls konstante, restliche Bruchteil die Kette in gewohnter Weise weiterführt.

Vorliegende Arbeit wurde durch Mittel der U.S.A. Air Force unterstützt. Grant AF-AFOSR 1446-68, Air Force Office of Scientific Research of the Office of Airspace Research.

4 J. M. Heras, A. J. Arvía, P. J. Aymonino und H. J. Schumacher, Z. physik. Chem. Neue Folge 28 (1961) 250. 